Revue des patrimoines

\title{
Les peintures murales de Saint-Pierre de Colonzelle : une découverte récente dans le canton de Grignan (Drôme)
}

\section{Geneviève Jourdan}

\section{OpenEdition}

Journals

Édition électronique

URL : https://journals.openedition.org/insitu/1240

DOI : 10.4000/insitu. 1240

ISSN : 1630-7305

Éditeur

Ministère de la Culture

\section{Référence électronique}

Geneviève Jourdan, «Les peintures murales de Saint-Pierre de Colonzelle : une découverte récente dans le canton de Grignan (Drôme) », In Situ [En ligne], 2 | 2002, mis en ligne le 23 avril 2012, consulté le 21 septembre 2021. URL : http://journals.openedition.org/insitu/1240 ; DOI : https://doi.org/ 10.4000/insitu. 1240

Ce document a été généré automatiquement le 21 septembre 2021.

\section{cc) $(1) \ominus$}

In Situ Revues des patrimoines est mis à disposition selon les termes de la licence Creative Commons Attribution - Pas d'Utilisation Commerciale - Pas de Modification 4.0 International. 


\title{
Les peintures murales de Saint-Pierre de Colonzelle : une découverte récente dans le canton de Grignan (Drôme)
}

\author{
Geneviève Jourdan
}

1 Eglise d'un prieuré clunisien mentionné dès le $\mathrm{X}^{\mathrm{e}}$ siècle, la chapelle Saint-Pierre-ès-liens de Colonzelle est un petit édifice roman du XII ${ }^{e}$ siècle, isolé dans la plaine. Son plan très simple, à chevet semi-circulaire, n'a pas été modifié. La construction en petit appareil, aux pierres de parement hachurées ou à chevrons, est agrémentée de marques lapidaires et d'un sobre décor sculpté. Son intégrité lui a valu l'inscription à l'Inventaire supplémentaire des Monuments historiques en 1926.

2 Rien ne laissait soupçonner pourtant que cette chapelle recelait aussi, dans le chœur, un ensemble de peintures murales pratiquement intact. En effet, depuis le XIX ${ }^{e}$ siècle au moins, l'intérieur était régulièrement blanchi à la chaux, sans doute selon les ordonnances épiscopales qui, dans une église voisine, qualifiaient « $d$ 'indécentes » les peintures médiévales.

Dans les années 1990, un décollement partiel du badigeon dû à l'humidité avait mis au jour un médaillon peint, L'Apparition du Christ à Marie-Madeleine ${ }^{1}$. Cette découverte a incité la municipalité à programmer l'enlèvement de l'enduit. Réalisé en octobre 2000, le dégagement total des peintures murales a fait apparaître dans le chœur un décor médiéval exceptionnel, entièrement conservé.

4 La peinture est posée directement sur un simple lait de chaux constituant la couche d'apprêt et laissant visibles les traces de taille de la pierre. La gamme chromatique se restreint au blanc de chaux, au noir et aux ocres rouge et jaune, couleurs qui, mélangées, donnent des tons intermédiaires, rose orangé et bordeaux. 
Figure 1

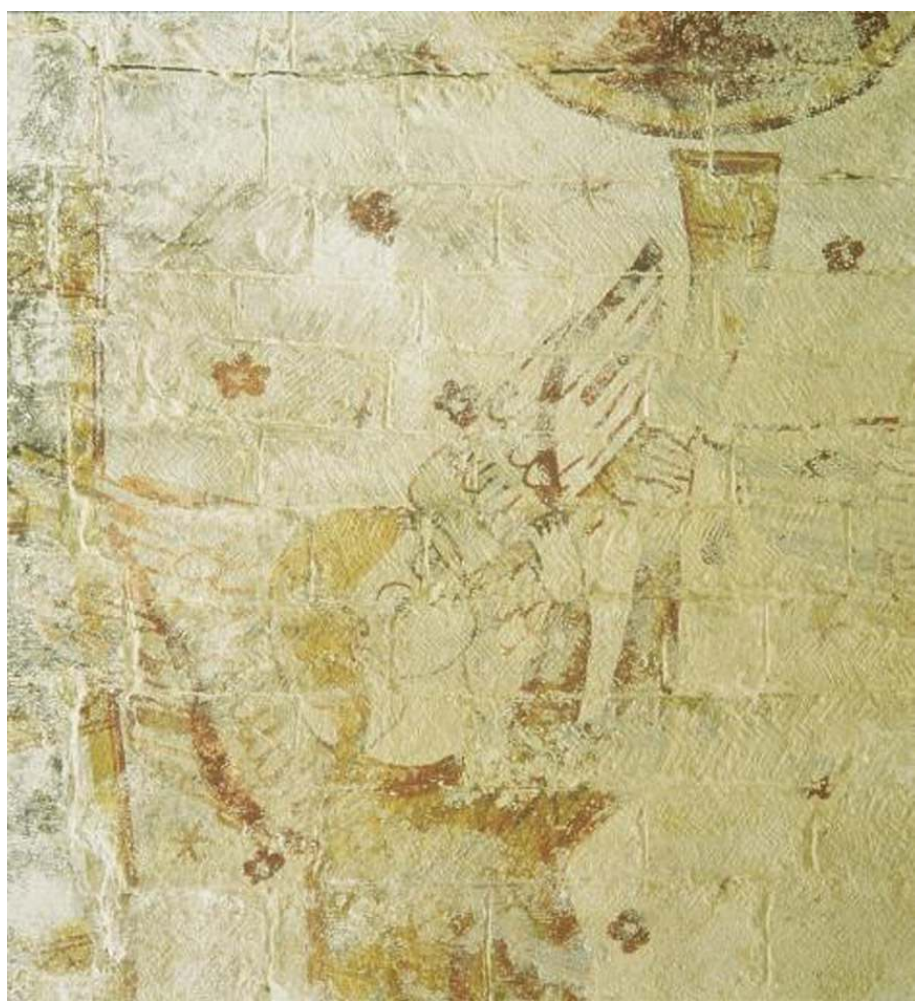

Travée de chœur, détail d'un ange sonnant de l'olifant

Phot. Inv. E. Dessert ( $)$ Inventaire général, ADAGP, 2001

5 Le style, plus graphique que pictural, est dépourvu de tout modelé : les sujets sont cernés d'un trait brun rouge, de grands à-plats colorent les surfaces, dans un jeu de teintes opposées donnant son unité à l'ensemble.

La composition s'organise en registres superposés. 
Figure 2

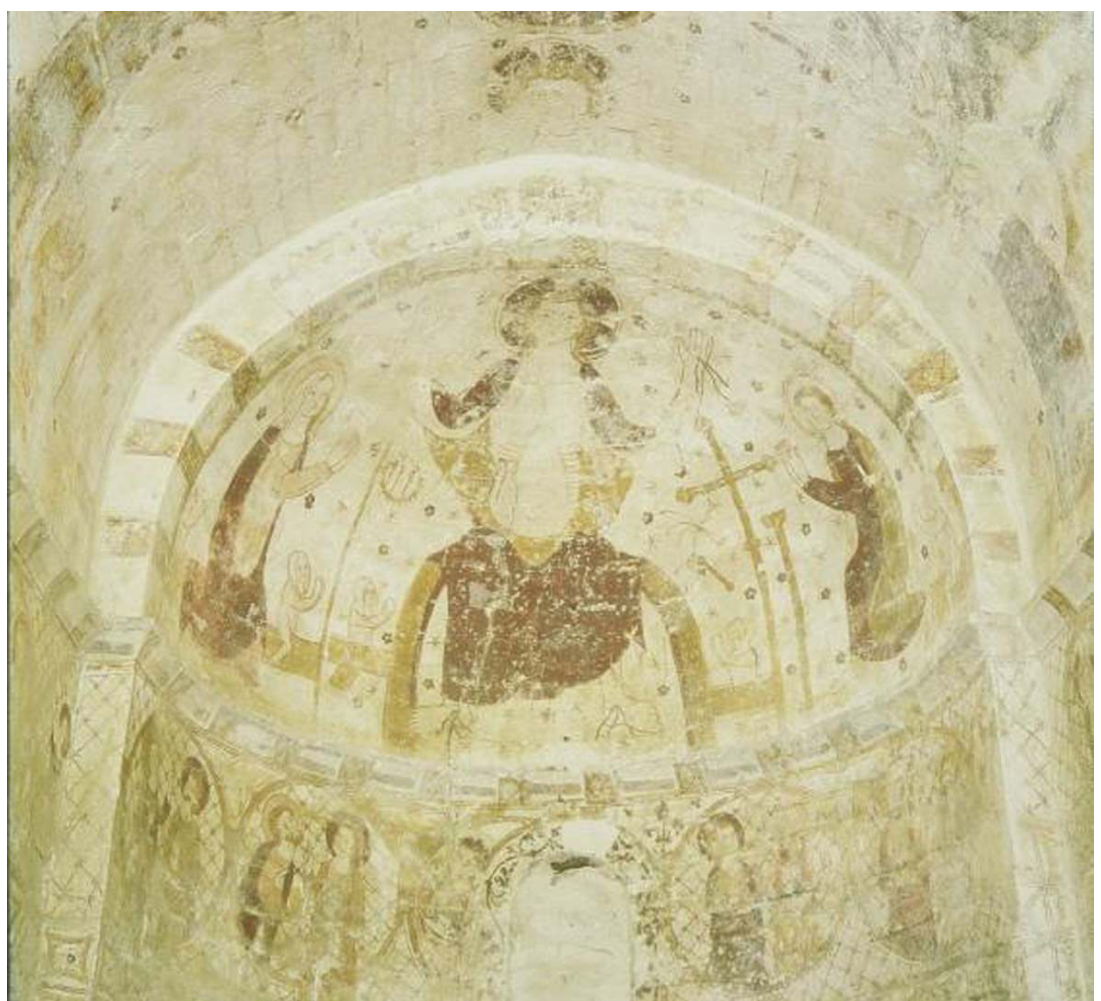

Vue d'ensemble de l'abside

Phot. Inv. E. Dessert ( $)$ Inventaire général, ADAGP, 2001

7 Un décor à motifs géométriques garnit la partie basse des murs, un bandeau de douze médaillons circulaires court sous la corniche, soulignant le sujet principal, la Résurrection des morts, traité sur les voûtes de la travée de chœur et de l'abside.

8 Le quadrillage du registre inférieur, où chaque carré est orné d'une fleurette centrale rouge à cinq lobes inscrite dans quatre segments de cercle jaunes, évoque soit des tentures, soit un faux appareil à double joint et à effet de bossage. 
Figure 3

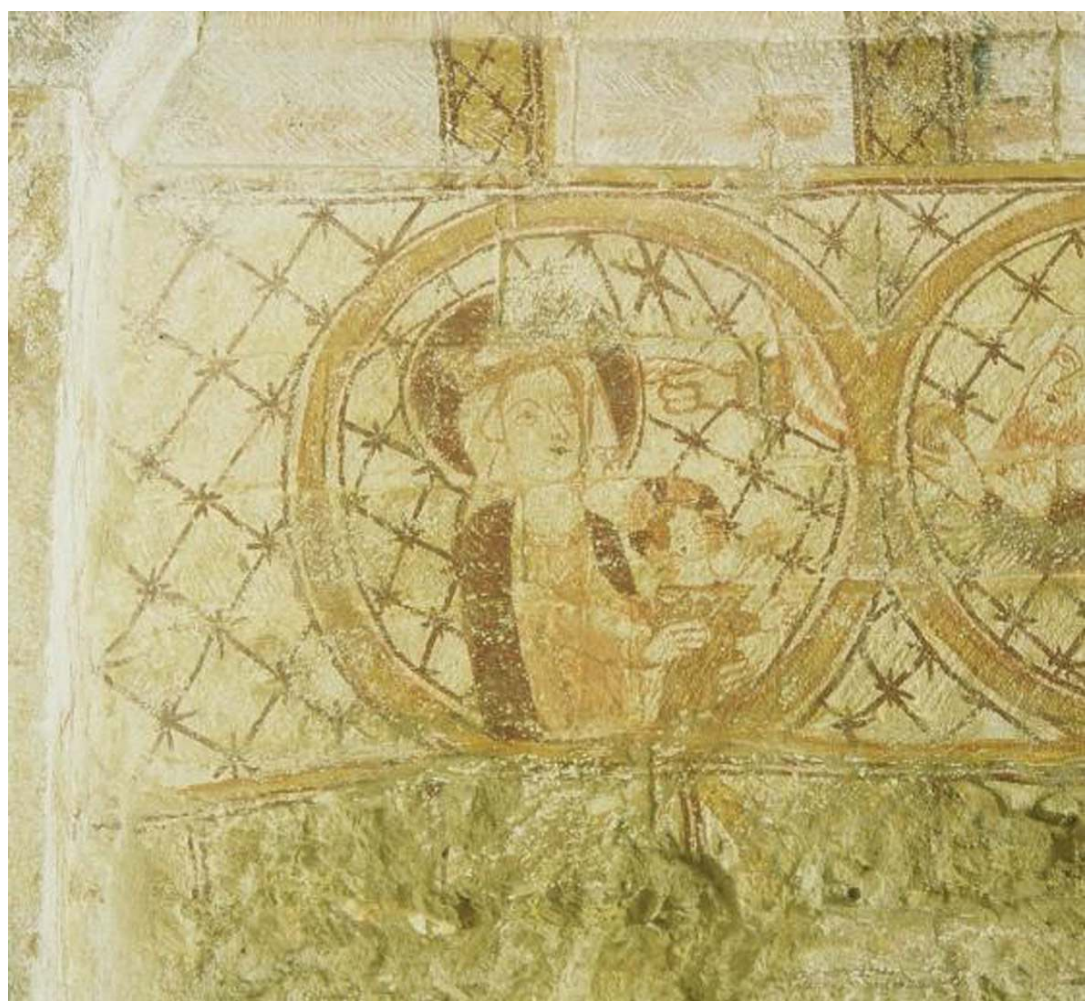

L'Adoration des mages, détail : la Vierge et l'Enfant en médaillon Phot. Inv. E. Dessert (C) Inventaire général, ADAGP, 2001

9 Les médaillons du registre médian présentent un ou deux personnages sur un fond réticulé. Dans la travée de chœur, à droite, se développe L'Adoration des Mages (fig. $\mathbf{n}^{\circ} \mathbf{3}$ ). L'Enfant Jésus, porté par sa mère dans le premier médaillon, est désigné par la main de Dieu et par l'étoile au-dessus de sa tête. 
Figure 4

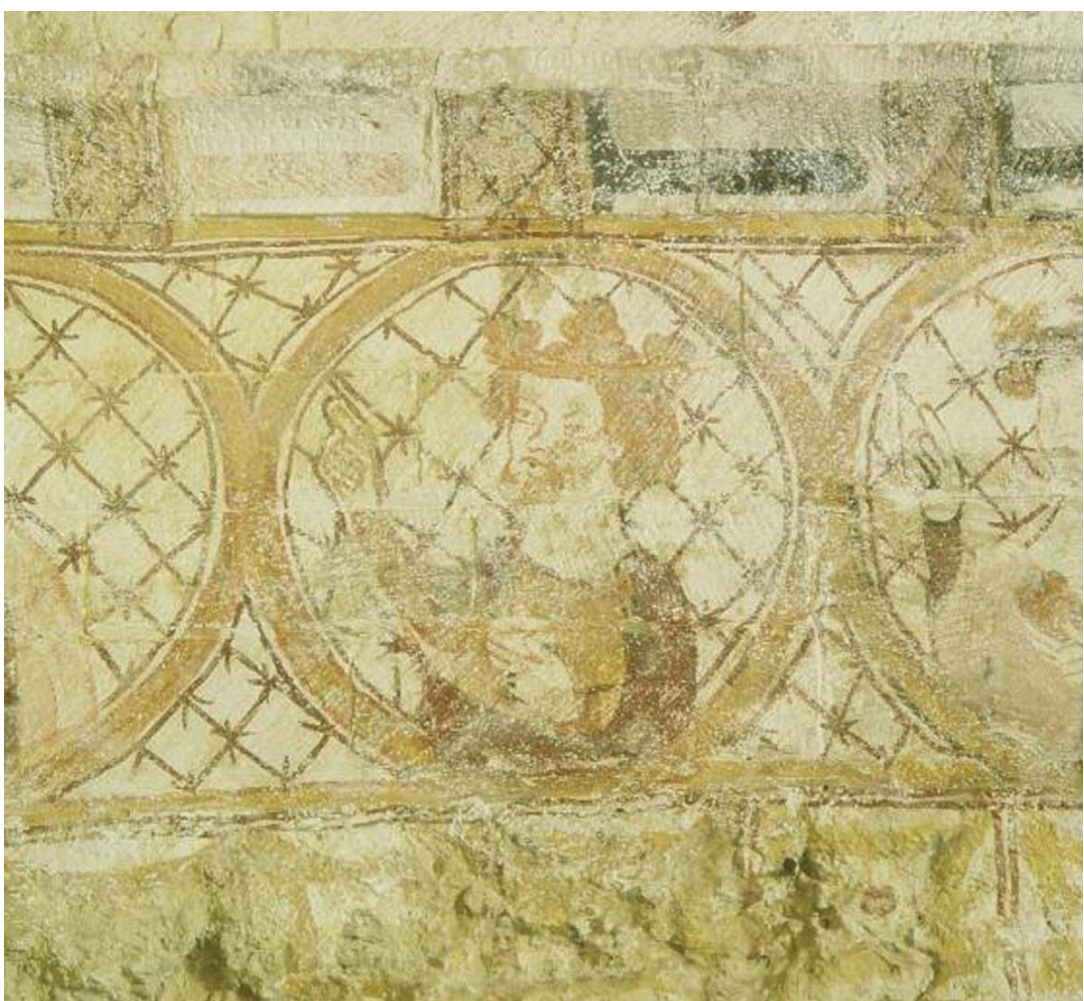

L'Adoration des mages, détail : le second roi mage en médaillon Phot. Inv. E. Dessert ( $@$ Inventaire général, ADAGP, 2001

10 Les mages, en buste, occupent chacun un médaillon (fig. $\mathbf{n}^{\circ} \mathbf{4}$ ); le premier n'est pas couronné, les deux autres portent une couronne à fleurons. 
Figure 5

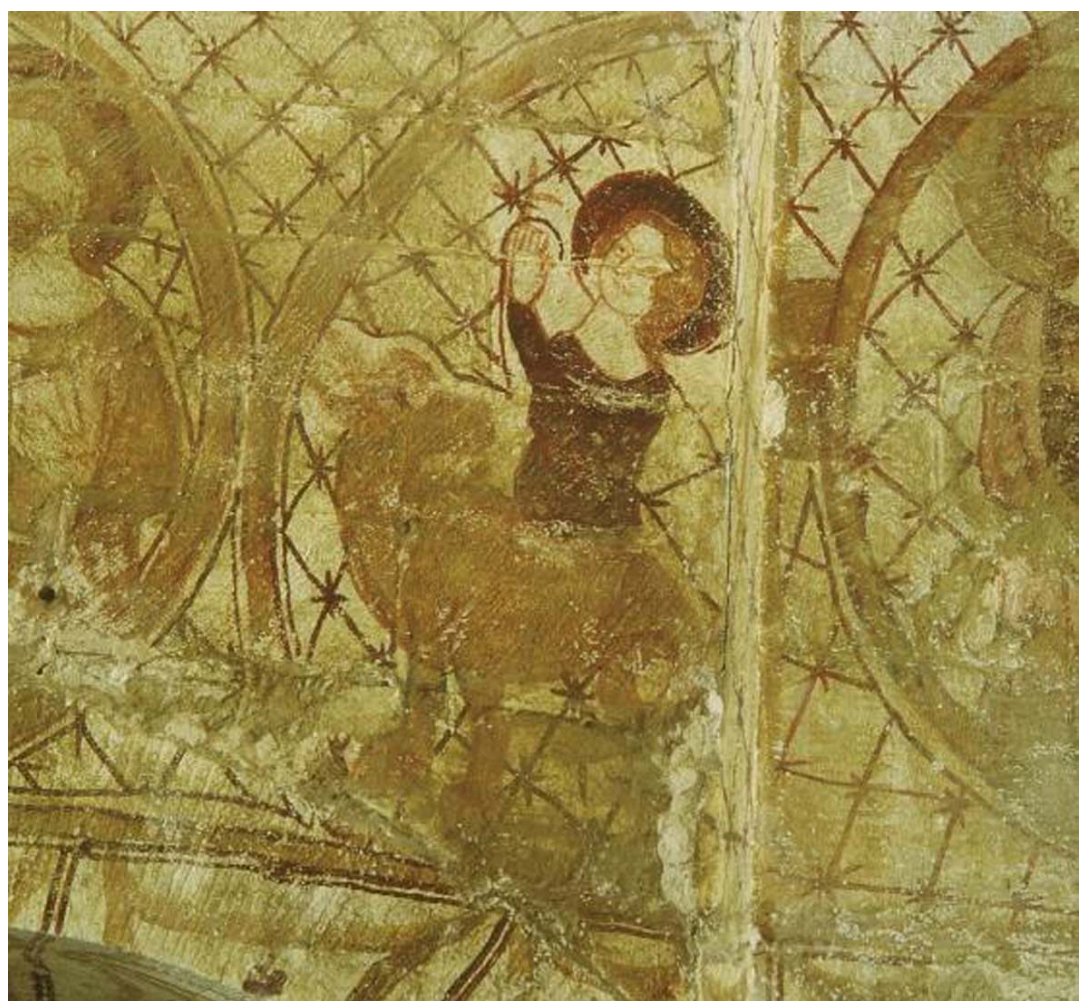

Le prophète Daniel dans la fosse aux lions

Phot. Inv. E. Dessert @ Inventaire général, ADAGP, 2001

11 A gauche font face trois médaillons, l'un à deux personnages (apôtres), les deux autres historiés: le Christ vivant apparaît à Marie-Madeleine et le Prophète Daniel dans la fosse aux lions (fig. $\mathbf{n}^{\circ}$ 5), image de l'enfer ; cette scène empruntée à l'Ancien Testament préfigure le Christ ressuscité et symbolise l'âme sauvée du mal. Ces deux sujets expriment fortement la résurrection du Christ et, avec les mages qui reconnaissent le Sauveur du monde, annoncent le thème représenté sur les voûtes, semées de fleurs et d'étoiles.

12 Au centre de l'abside trône l'immense figure du Christ-juge (fig. $\mathbf{n}^{\circ} \mathbf{6}^{\mathbf{6}}$ ) en majesté, bras levés, poitrine découverte, montrant les plaies de sa crucifixion que rappellent à ses côtés les instruments de la Passion; de part et d'autre, agenouillés en prière, la Vierge et saint Jean l'Evangéliste intercèdent en faveur des pécheurs (fig. $\mathbf{n}^{\circ} 7$ ), tandis qu'à leurs pieds les humains sortent de leurs tombeaux, les mains jointes et le regard levé vers le Christ (fig. $\mathbf{n}^{\circ} \mathbf{8}$ ). Ce thème illustre le premier instant du Jugement dernier. 


\section{Figure 6}

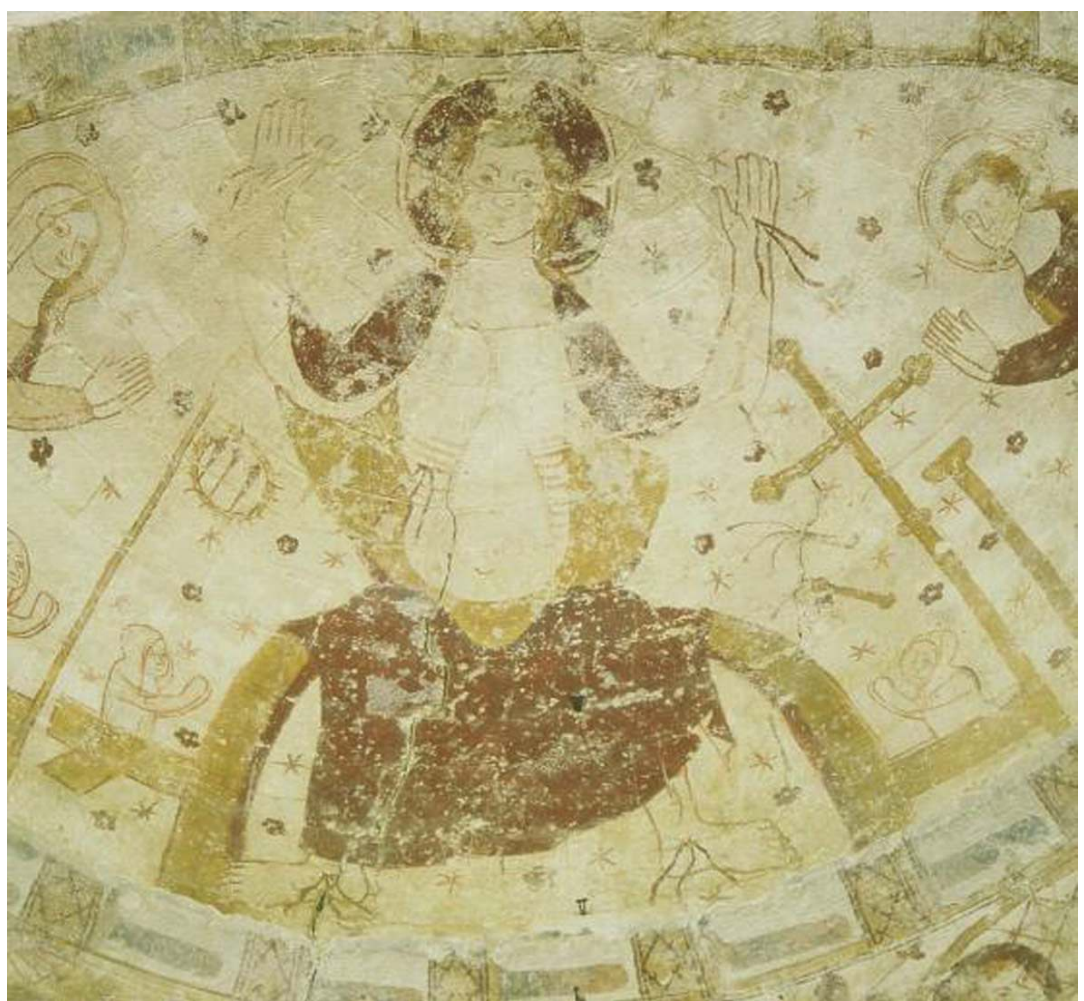

Le Christ-juge, montrant les plaies de sa crucifixion

Phot. Inv. E. Dessert (C) Inventaire général, ADAGP, 2001 
Figure 7

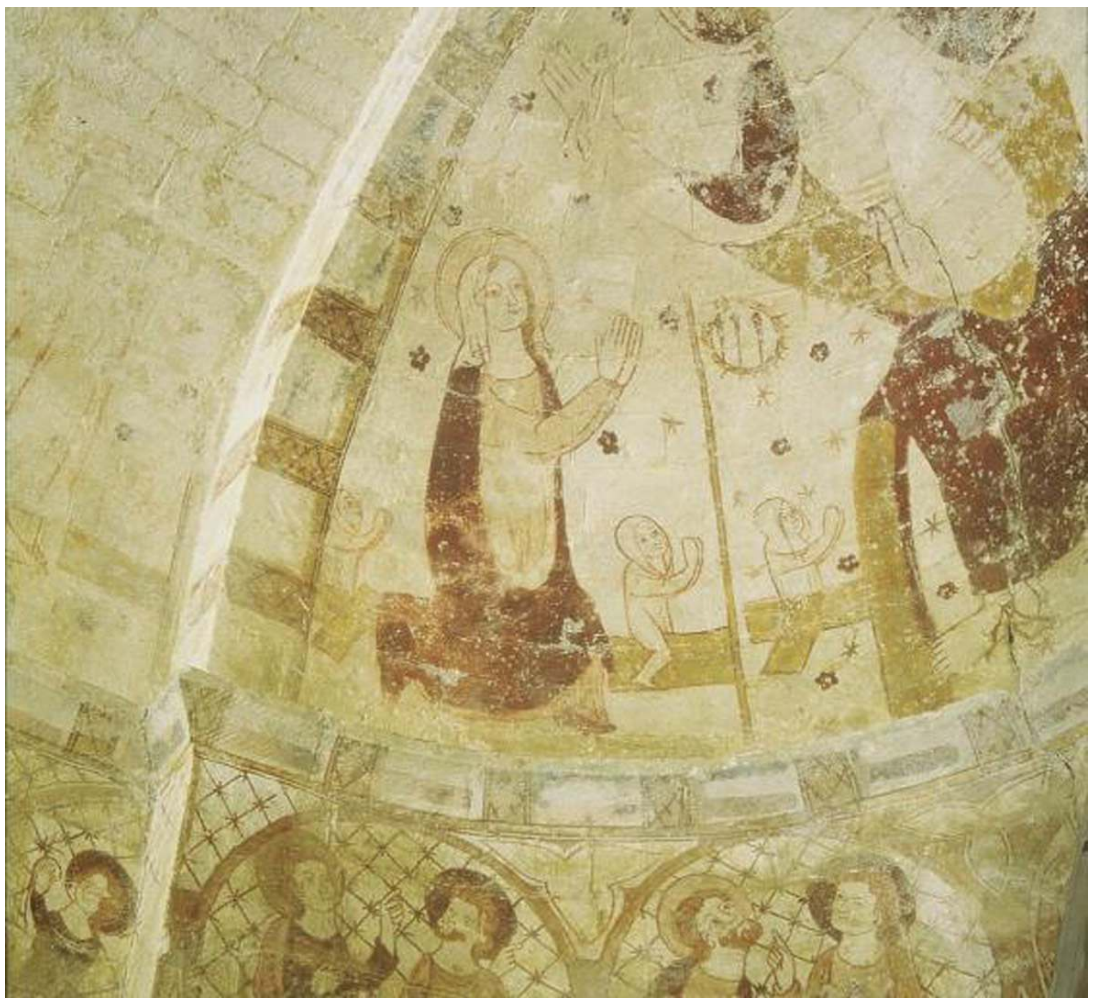

La Vierge en prière, intercédant en faveur des pêcheurs Phot. Inv. E. Dessert () Inventaire général, ADAGP, 2001 
Figure 8

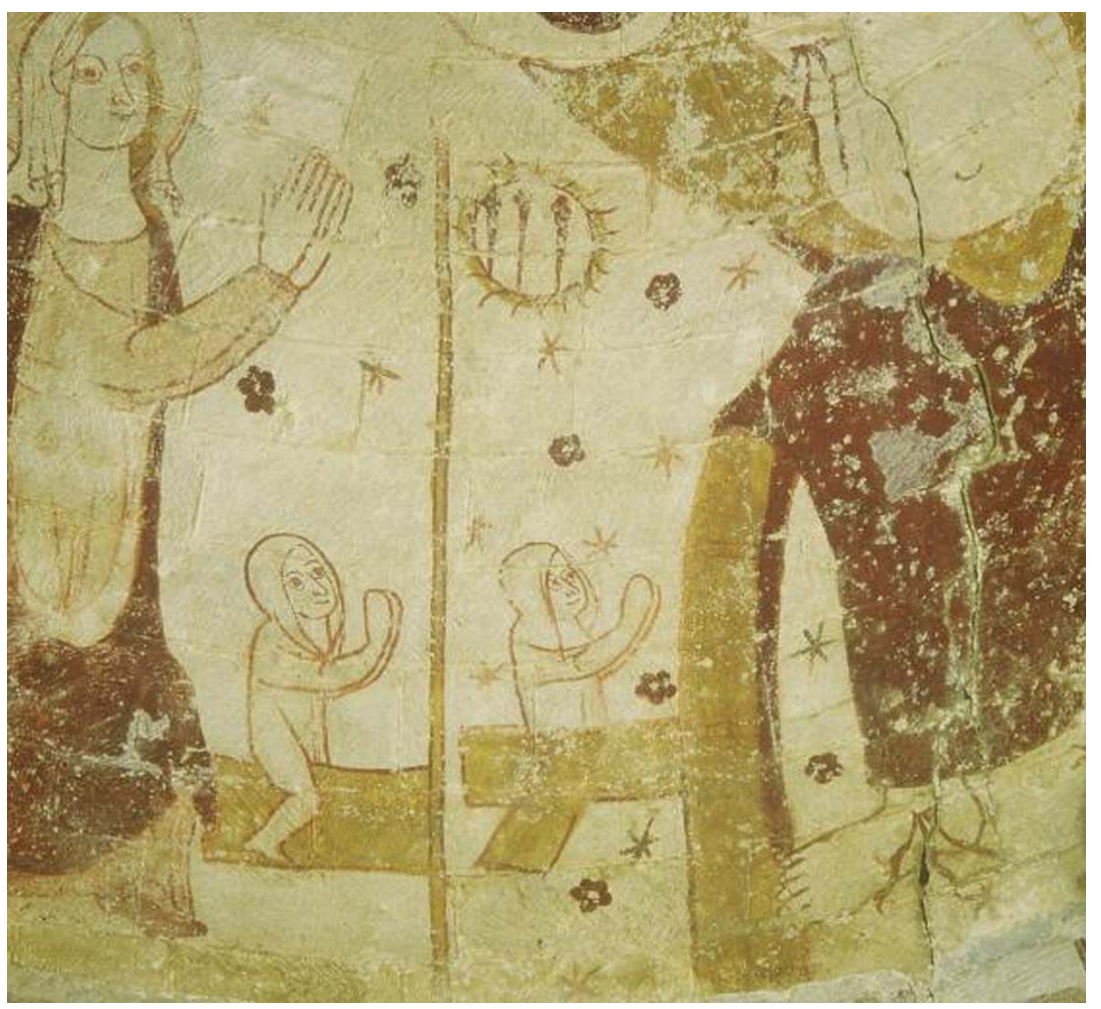

Abside, détail : les morts ressuscitent et sortent de leurs tombeaux

Phot. Inv. E. Dessert ( $)$ Inventaire général, ADAGP, 2001 


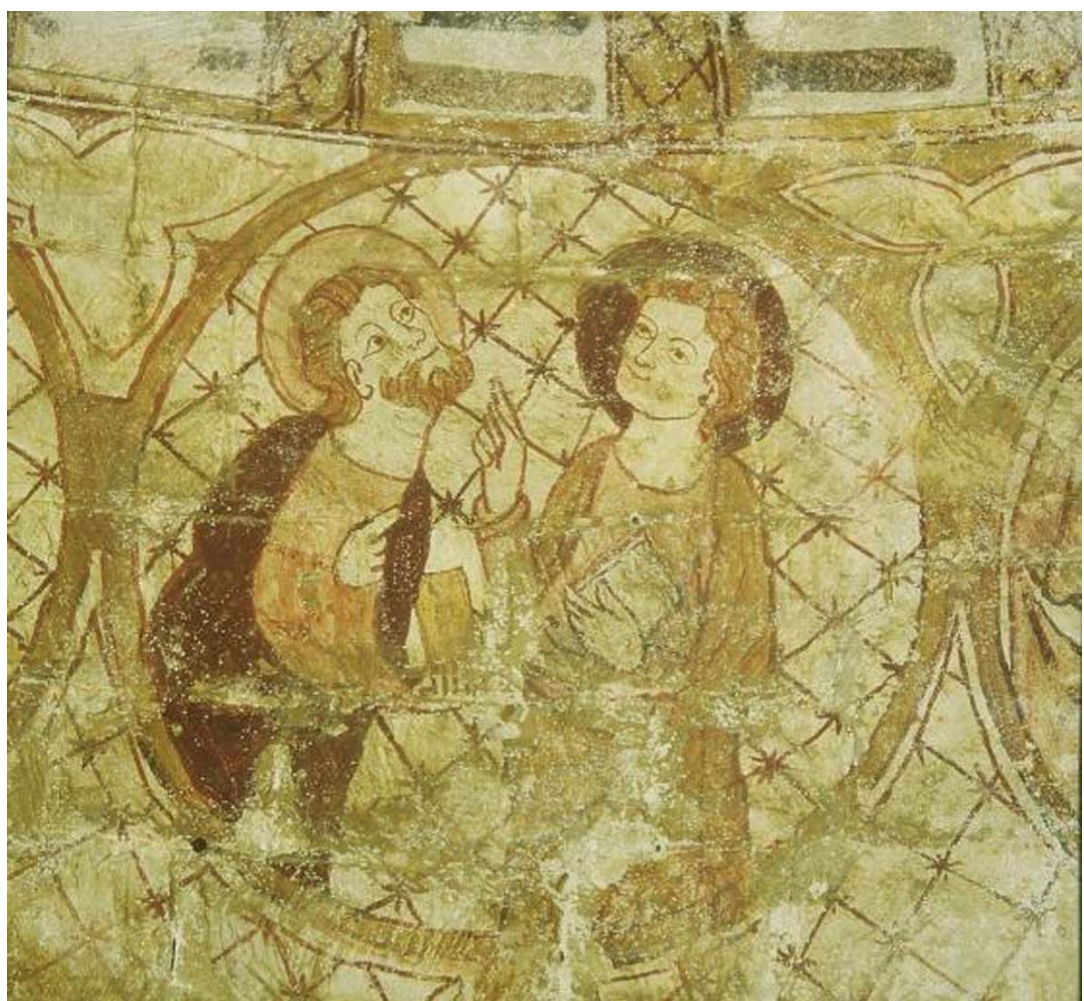

Bandeau médian de l'abside, détail : deux apôtres en médaillon

Phot. Inv. E. Dessert ( $)$ Inventaire général, ADAGP, 2001

Au-dessous, les apôtres, figurés deux par deux dans les médaillons situés sous la corniche, participent à la scène en tant qu'assesseurs de la Vierge et de saint Jean. Les morts, représentés tout petits et encore enveloppés de leurs linceuls, ressuscitent en même temps, surgissant de toutes parts au signal donné par les anges, qui sonnent de l'olifant de chaque côté de la voûte de la travée de chœur (fig. $\mathbf{n}^{\circ}$ 9). L'ensemble de l'iconographie converge au sommet de cette voûte, autour d'un visage du Christ au nimbe crucifère et de l'Agneau immolé, en médaillon, évoquant l'attente du Jugement.

Dans le registre médian, l'un des médaillons étant coupé par l'arête du mur, on remarque que les apôtres sont au nombre de onze seulement, puisque saint Jean est déjà représenté dans le cul-de-four de l'abside. Mais près de la fenêtre axiale murée, à l'encadrement entouré d'un rinceau de feuillage, un personnage non nimbé et coiffé d'une sorte de feuille, semble se cacher dans un médaillon tronqué : ne pourrait-il s'agir de Judas, l'un des apôtres?

Par son style et son iconographie, ce décor est datable du tout début du XIV ${ }^{e}$ siècle. Au thème byzantin du Jugement dernier, l'Occident a apporté, dès la fin du XII ${ }^{e}$ siècle, des mutations iconographiques ici présentes: le Christ-juge n'est plus terrifiant, sous sa forme apocalyptique, avec une épée dans la bouche, mais figuré sous sa forme évangélique, en rédempteur ; à ses côtés, l'apôtre saint Jean remplace saint Jean-Baptiste le précurseur. Enfin, aucune allusion n'est faite à l'Apocalypse. 
Figure 10

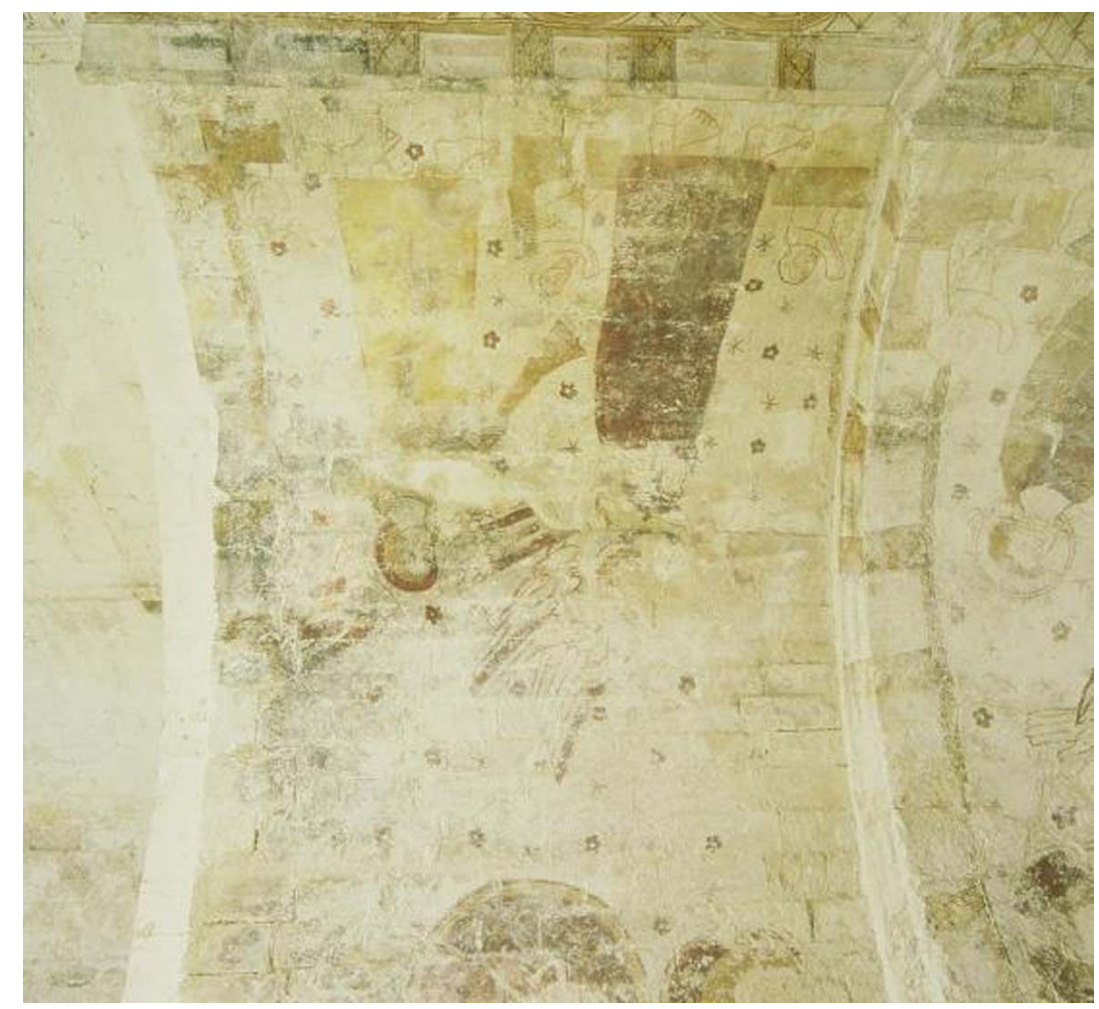

Travée de chœur : anges sonnant de l'olifant

Phot. Inv. E. Dessert (C) Inventaire général, ADAGP, 2001

Ce programme iconographique est unique en Drôme provençale. Si le sujet du Jugement dernier existe dans la peinture médiévale en Rhône-Alpes, celui de la Résurrection des morts se rencontre moins fréquemment. Il apparaît cependant dans la chapelle Saint-Paul de Lacenas ${ }^{2}$ (Rhône) : au revers de l'arc triomphal et à la naissance de la voûte de la travée de chœur, la composition de la résurrection des morts est assez semblable à celle des peintures de Colonzelle (fig. $\mathbf{n}^{\circ} \mathbf{1 0}$ ); dans l'ensemble du décor de cette chapelle, daté de la fin du XIII ${ }^{e}$ siècle ou du tout début du XIV ${ }^{e}$, une représentation des visages et des corps similaires, des anges en médaillons, un fond semé d'étoiles, ajoutent à la relation entre les deux œuvres. Un rapprochement stylistique peut être également fait avec les personnages des peintures profanes du château de Verdon Dessous à Cruet (Savoie, déposées au musée de Chambéry) ${ }^{3}$, contemporaines, dont la facture, très voisine, semble issue d'un même courant artistique. 


\section{NOTES}

1. Tardieu, Joëlle. Drôme. Colonzelle. Eglise Saint-Pierre. In : Peintures murales médiévales des églises de Rhône-Alpes. Art et Archéol. Rhône-Alpes. Cah. de René de Lucinge, $\mathrm{n}^{\circ}$ spécial 7. Lyon : 1998, p. 53-54.

2. Meras, Mathieu. Les peintures murales de la chapelle Saint-Paul. B.A.V.B., 1979-1980, p. 28-31.

Peyrelongue, A. et Lavigne, M. Les peintures médiévales de Notre-Dame du Sou (Rhône). Archéologia, n 151, février 1981, p. 54-60.

Belle, Véronique. Inventaire des églises et chapelles du canton de Villefranche-sur-Saône (Rhône). Mémoire de maîtrise d'histoire de l'art. Université Paris I, 1988-1989, vol. 1, p. 73-77, vol. 2, fig. 141-144.

3. Raffaelli, Philippe. Cruet (Savoie). Château de Verdon Dessous. 1280-1310. In : Fresques et peintures murales en pays de Savoie. Soc. Savoisienne d'Hist. et d'Archéol., 1988, p. 84-87.

INDEX

Mots-clés : inventaire général, en ligne, journal, revue électronique, revue numérique, périodique, patrimoine, histoire de l'art, France, Rhône-Alpes, peinture murale, 13e siècle, 14e siècle

Keywords : on line, electronic journal, heritage, history of art, mural paintings, XIIIth century, XIVth century

\section{AUTEUR}

\section{GENEVIÈVE JOURDAN}

Conservateur du patrimoine, Service régional de l'Inventaire, DRAC du Rhône-Alpes Le Grenier d'Abondance 6, quai Saint-Vincent 69283 Lyon Cédex 01. genevieve.jourdan@culture.gouv.fr 